\title{
Failure to learn in a taste aversion paradigm: Associative or performance deficit?
}

\author{
DAVID C. RICCIO and VAHRAM HAROUTUNIAN \\ Kent State University, Kent, Ohio 44242
}

\begin{abstract}
A transfer paradigm was employed to examine the possibility that the apparent failure of rats to associate exteroceptive stimuli with internal malaise reflects a performance deficit. Repeated pairings of distinctive apparatus cues with rotation-induced motion sickness produced no evidence of conditioned suppression to the CS complex. However, relative to controls which had received noncontingent rotational experience, the experimental rats acquired conditioned fear more rapidly when the apparatus cues were paired with footshock in the transfer phase. This facilitation of conditioning suggests that an associative representation between exteroceptive stimuli and internal consequences was acquired but not expressed in overt responding.
\end{abstract}

A variety of studies indicate that rats can readily acquire taste aversions when gustatory cues are paired with internal malaise, but often fail to evidence learning when exteroceptive stimuli are paired with the same internal consequences (e.g., Garcia \& Koelling, 1966; Revusky, 1971). Conversely, acquisition of conditioned responses can occur quickly if external cues predict painful events, while conditioning may occur slowly or not at all with a similar relationship between taste cues and noxious stimulation (e.g., Domjan \& Wilson, 1972; Garcia \& Koelling, 1966). These findings, sometimes collectively referred to as the "Garcia effect," have provided an important challenge to the traditional proposition that, within broad limits, stimuli are equivalent and interchangeable in conditioning (Revusky \& Garcia, 1970; Seligman, 1970). Although there are in fact some studies showing that rats can learn to avoid a spatial location associated with poisoning (Best, Best, \& Mickley, 1973; Rozin, 1969), these findings have generally been taken as evidence of a relative rather than an absolute dichotomy of associable classes of stimuli. Consequently, while some investigators have emphasized the relevance or salience of various stimuli (e.g., Revusky, 1971) and others the organism's preparedness to associate the relevant stimulus events (Seligman, 1970), there seems to be substantial agreement that the phenomena reflect differential associability in the learning process.

While the current emphasis on the modulation of learning by the biological characteristics of the organism is undoubtedly an important corrective position, further analysis of these associative deficits appears warranted. In particular, the possibility that the

The assistance of Chris Papantonakis, Kim Kovacs, and Heather Schroeder in the collection of data is gratefully acknowledged. Reprint requests should be sent to David C. Riccio, Department of Psychology, Kent State University, Kent, Ohio 44242. measures of performance may inadequately reflect the extent of learning actually achieved has been overlooked.

Consider, for example, a finding from our lab which is quite congruent with the "Garcia effect." Rats given saccharin-flavored water and then made motion sick through exposure to rotation rapidly developed a strong taste aversion, even when the CS-UCS interval extended over minutes. In contrast, repeated pairings of distinctive external cues with comparable motion sickness treatment failed to elicit conditioned suppression in a CER paradigm. Moreover, the repeated application of rotation as a punishing stimulus in an instrumental contingency was also unsuccessful in producing passive avoidance, whereas mild footshock in the same situation typically yields passive avoidance in a single trial (Haroutunian \& Riccio, 1975).

Despite the consistent tenor of the data, it remains possible that, in the latter two instances, the behavioral response required may have been insensitive or inappropriate to the associative process. That is, the venerable distinction between learning and performance might well be of relevance in this paradigm. In this respect, Rescorla and Holland (1976) have cogently noted that CSs may differ not only in their associability with a UCS, "but in their ability to evoke behavior based upon equal associations" (p. 182).

If learning occurs but is not reflected in concurrent performance when distal stimuli are paired with malaise, then the associative status of these events might more sensitively be determined by using a transfer paradigm. Facilitation of acquisition when the original CS is re-paired with moderate shock in the transfer phase would suggest that learning had indeed occurred in the original training. The following experiment evaluated such a possibility, utilizing a conditioned suppression paradigm and controls for noncontingent effects of rotational exposure. 


\section{METHOD}

\section{Subjects}

Twenty naive male albino rats were used in this experiment. All subjects were housed in single wire-mesh cages. Except for the first day of the experiment, when no water was available, the rats were maintained on ad-lib food and water throughout the experiment.

\begin{abstract}
Apparatus
A $20 \times 20 \times 19 \mathrm{~cm}$ black Plexiglas box with a grid floor was used as the conditioning chamber. The grid floor was constructed of $.3-\mathrm{cm}$ stainless steel rods spaced $2 \mathrm{~cm}$ apart. The terminals of the grid floor could be connected to either a shock scrambler (Foringer, $1925 \mathrm{M} 111)$, which delivered a 2-sec 150-V (1.0-mA) shock, or to one pole of a Grason-Stadler drinkometer circuit. A $1-\mathrm{cm}$ hole was cut $3 \mathrm{~cm}$ above the floor on one wall of the chamber to allow a metal drinking spout to be inserted into the chamber. This drinking spout was connected to the second pole of the drinkometer circuit. A 50:50 solution of Eagle Brand sweetened condensed milk and water was available through the drinking spout. A turntable set to rotate at $70 \mathrm{rpm}$ was also used. Four equally spaced clamps were mounted around the perimeter of the turntable. These clamps were used to secure either the experimental chamber or the home cage to the turntable during rotation.
\end{abstract}

\section{Procedure}

Following $24 \mathrm{~h}$ of water deprivation, all subjects were given access to the sweetened milk solution for $5 \mathrm{~h}$. For the remainder of the experiment the rats had ad-lib access to both food and water, with the exception of a total of approximately $1 \mathrm{~h}$ per day during which they received the experimental manipulations.

For the 8 days following the initial exposure to sweetened milk, each rat assigned to the experimental (E) group $(\mathrm{N}=8)$ was placed inside the black experimental chamber. The chamber, mounted on the turntable, was rotated for $301 / 2$-min periods at $70 \mathrm{rpm}$, with an interrotation interval of $2.5 \mathrm{sec}$. The rat was then returned to its home cage; $1 \mathrm{~h}$ later the water bottle was replaced with one containing $10 \mathrm{ml}$ of the sweetened milk solution, which was invariably consumed within $20 \mathrm{~min}$. Once the $10 \mathrm{ml}$ of milk was consumed, the water bottle was returned. Eight rats were assigned to Group $\mathrm{C}_{1}$ and served as controls for nonassociative consequences of rotation and exposure to the apparatus cues. These rats were confined individually inside the black chamber for $15 \mathrm{~min}$ but were not rotated. This group was exposed to rotation in their home cages not less than $1 \mathrm{~h}$ following their confinement in the black box. One hour after the rotation treatment, these rats were given access to $10 \mathrm{ml}$ of the sweetened milk solution. To control for possible latentinhibition-type effects of confinement in the black box in the absence of rotation, an additional smaller group $(N=4)$ was employed (Group $C_{2}$ ). These animals also received rotation in their home cages, followed $1 \mathrm{~h}$ later by presentation of the $10 \mathrm{ml}$ of sweetened milk, but were never exposed to the black chamber.

In the second phase of the experiment, a conditioned suppression transfer test was used to assess the effects of pairing rotation with the black chamber cues in Phase 1. All rats were tested for suppression of drinking sweetened milk in the black box. This was followed $1 \mathrm{~h}$ later by a reconditioning session where shocks were paired with the black box apparatus cues. This two-step procedure insured that the suppression scores on any given day were not affected by the unconditioned consequences of shock. Each day, for 6 consecutive days, each rat was placed inside the black box, given access to sweetened milk, and its latency to begin drinking was recorded. Drinking was defined as 10 licks from the metal spout. Once drinking occurred, the rat was returned to its home cage, where the remainder of the sweetened milk was available. Each rat was then returned to the black chamber $1 \mathrm{~h}$ later for a 3-min confinement period. During this time, one $2-\mathrm{sec} 150-\mathrm{V}(1.0-\mathrm{mA})$ scrambled noncontingent shock was delivered. The time interval between placement in the box and administration of shock was varied on a quasirandom basis. The rat was returned to its home cage at the end of the 3 -min confinement period. This procedure was repeated once a day for 6 consecutive days.

\section{RESULTS}

Median latencies to begin drinking during test sessions for all three groups are presented in Figure 1. The three groups did not differ significantly on the first drinking latency measure taken in Phase 2 (Kruskal-Wallis nonparametric analysis of variance, $\mathrm{H}=1.86, \mathrm{p}>.05$ ). (It should be noted that Day 1 latencies could not have been influenced by the shock treatments, as the measure was obtained prior to the delivery of any shocks.) The differences in the latencies to begin drinking for the three groups did not reach significance $(\mathrm{Hs} \leqslant 4.50$, ps $>.05$ ) until Days 5 and 6 of Phase 2, at which time there was a significant effect of treatment conditions $(\mathrm{H}=6.9, \mathrm{p}<.05$ and $\mathrm{H}=8.4, \mathrm{p}<.02$, respectively $)$. Individual Mann-Whitney $U$ comparisons of drinking latencies on Days 5 and 6 revealed that the rats in the experimental group took significantly longer to begin drinking that those in the two control groups $(p<.05)$, which did not differ from each other ( $p s>.05$ ). Furthermore, Friedman's two-way analysis of variance showed that the drinking latencies of Groups $C_{1}$ and $C_{2}$ did not change significantly as a function of treatment days $\left(\mathrm{X}_{\mathrm{r}}^{2} \mathrm{~s}<8.6\right.$, ps $\left.>.05\right)$. The latencies to begin drinking for the experimental group, however, did increase significantly as a function of treatment days $\left(X_{\mathrm{r}}^{2} \mathrm{~s}=23.45, \mathrm{p}<.001\right)$.

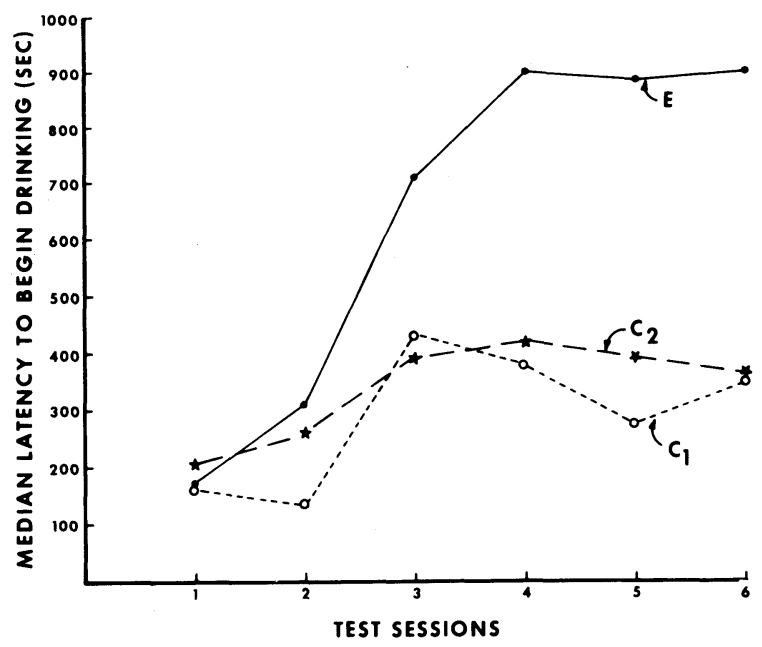

Figure 1. Median latencies to begin drinking, following the rotational treatment phase, for the experimental (E) and the two control groups $\left(C_{1}\right.$ and $\left.C_{2}\right)$. Each test session was followed by a CS-shock pairing; thus, the scores of Session 1 reflect the effects of rotation treatments prior to any CS-shock pairings. 


\section{DISCUSSION}

The data demonstrate that pairing of exteroceptive cues with rotation-induced illness can facilitate subsequent acquisition of shock-induced suppression to the same CS. An important feature of the findings is that, prior to introduction of shock, there was no discernible indication that learning had occurred: On the initial trial of Phase 2 the latency of the experimental group to begin drinking was almost identical to that of controls. Thus, in the absence of the transfer test, the data might readily be interpreted as supporting the view that an associative deficit exists when these different classes of stimuli are paired.

The positive transfer to the conditioned suppression task in the experimental animals is not readily attributable to the summative effect of aversive treatments, as both groups (experimental and $C_{1}$ controls) received comparable rotational experience. In the control groups, however, illness was not contingent upon prior cue presentation. On the basis of performance in Control Group 1, it might be argued that the differential learning reflects impaired acquisition by controls due to a latent-inhibition-type effect. That is, the prior uneventful exposure to the black compartment, while intended to equate stimulus exposures, might reduce the subject's attention to, or processing of, the relevant CS when shock was introduced in Phase 2, thus interfering with acquisition of the CER (e.g., Carlton \& Vogel, 1967; Lubow, 1973). This concern seems obviated, however, by the fact that Control Group 2, which lacked preexposure to the CS in Phase 1 , showed nearly identical performance to Group $C_{1}$. Of perhaps greater concern in accounting for the differential transfer is the possibility that exposure to noncontingent illness in the controls served to depress their later acquisition of conditioned suppression. Although preexposure to illness has been shown to impair learning of a sickness-induced taste aversion (e.g., Braveman, 1975), it is not clear that prior illness can modify later learning with shock as the aversive agent. Indeed, a recent study in this lab confirmed the preexposure effect with rotation-induced illness, but failed to find retarded acquisition when sickness was produced by a different agent (e.g., LiCl) in the taste aversion phase (Haroutunian, Riccio, \& Gans, 1976). Thus, it is unlikely that prior experience with rotation retarded the acquisition of shock-induced response suppression in the two control groups of the present study. The lack of suppression seen in these controls is more likely related to the fact that, on the basis of pilot work, shock values were selected which would not produce complete and immediate cessation of responding.

One important issue not addressed here concerns the generality of these findings, especially with respect to more traditional illness-inducing agents as the UCS. For example, it might be argued that rotation-induced sickness involves exteroceptive as well as interoceptive aspects of aversiveness. Indeed, it was this reasoning that motivated our earlier attempt to condition external cues to a motion sickness response (Haroutunian \& Riccio, 1975). Accordingly, it should be emphasized that in that study, as well as in Phase 1 of the present experiment, our direct measures failed to indicate learning to contextual cues. In this respect, then, rotation seems comparable to more traditional sickness-inducing agents. Nevertheless, it remains important to determine whether an agent such as $\mathrm{LiCl}$ will also yield similar evidence of associative linkages to external stimuli when tested in a transfer paradigm. The converse side of the picture also needs to be explored: To what extent, if any, are latent associations acquired in tasks where gustatory cues paired with painful events appear to be ineffective?

The present findings, while based upon a paradigm very different from that of traditional latent learning studies (Tolman \& Honzik, 1930), are in agreement with the general interpretation of those earlier studies: Associations which are acquired need not be mirrored by performance changes. Recently, Best et al. (1973) have shown that, while rats can acquire an aversion to visual stimuli of a distinctive compartment, the effect is appreciably increased if the animals are not given an opportunity to drink flavored water while in the presence of the exteroceptive cues. The authors suggest that, in the absence of easily associable cues, the animals can learn the relationship between other (external) stimuli and malaise. Our data extend this notion by demonstrating that, following cue-malaise pairings, some representation is established which, even when not expressed overtly to the CS, can enhance subsequent fear conditioning. This evidence should not be viewed as detracting from the recent emphasis on the role of biological contributions and constraints on the learning process. It does suggest, however, that appropriate caution needs to be exercised with respect to interpretations of learning deficits.

Finally, further evidence of the formation of covert associations in Pavlovian conditioning is provided by two studies which appeared after the present paper was substantially completed. For example, Rudy, Iwens, and Best (1977) report that pairing novel external cues with illness attenuates the strength of a subsequent illness-induced taste aversion. Apparently, an association between novel stimuli and the illness UCS was acquired which interfered with the effectiveness of taste as a CS. In addition, Holland (1977) has presented an extensive analysis of the relation between the type of CS and the form of the CR. He demonstrates that the same UCS in a Pavlovian arrangement may result in very different CRs, depending upon the nature of the CS. Although Holland's study employed food as the UCS, the findings, as the author notes, imply that deficits in response production must be considered seriously in evaluating the outcome of exteroceptive CS and illness pairings. To the proposal that blocking and second-order conditioning may provide alternative techniques for assessing the associations to a CS (Holland, 1977), we would add that the relatively simple transfer paradigm used here may also prove informative.

\section{REFERENCES}

Best, P. L., Best, M. R., \& Mickley, G. A. Conditioned aversion to distinct environmental stimuli resulting from gastrointestinal distress. Journal of Comparative and Physiological Psychology, 1973, 85, 250-257.

Braveman, N. S. Formation of taste aversion in rats following prior exposure to sickness. Learning and Motivation. 1975, 6. $512-534$

Carlton, P. L., \& Vogel, J. R. Habituation and conditioning. Journal of Comparative and Physiological Psychology, 1967, 63, 348-351.

Doman, M., \& Wilson, N. E. Specificity of cue to consequence in aversion learning in the rat. Psychonomic Science, 1972, 26, 143-145.

Garcia, J., \& Koelling, R. A. Relation of cue to consequence in avoidance learning. Psychonomic Science, 1966, 4, 123-124.

Haroutunian, V., \& Riccio, D. C. Acquisition of rotationinduced taste aversion as a function of drinking-treatment delay. Physiological Psychology, 1975, 3, 273-277.

Haroutunian, V., Riccio, D. C., \& Gans, D. Suppression of drinking following rotational stimulation as an index of motion sickness in the rat. Physiological Psychology, 1976, 4, 467-472.

Holland, P. C. Conditioned stimulus as a determinant of the form of the Pavlovian conditioned response. Journal of Experimental Psychology: Animal Behavior Processes, 1977, 3, 77-104.

Lubow, R. E. Latent inhibition. Psychological Bulletin, 1973, 79, 398-407.

Rescorla, R. A., \& Holland, P. C. Some behavioral approaches to the study of learning. In E. Bennett \& M. Rozenweig (Eds.), Neural mechanisms of learning and memory. Cambridge: MIT Press, 1976.

REVUSKY, S. H. The role of interference in association over a delay. In W. K. Honig \& H. James (Eds.), Animal memory. New York: Academic Press, 1971. 
Revusky, S. H., \& Garcia, J. Learned associations over long delays. In G. Bower \& J. T. Spence (Eds.), Psychology of learning and motivation (Vol. 4). New York: Academic Press, 1970.

Rozin, P. Central or peripheral mediation of learning with long CS-US intervals in the feeding system. Journal of Comparative and Physiological Psychology, 1969, 67, 421-429.

Rudy, J. W., Iwens, J., \& Best, P. J. Pairing novel exteroceptive cues and illness reduces illness-induced taste aversions. Journal of Experimental Psychology: Animal Behavior Processes, 1977. 3. $14-25$.
Seligman, M. E. P. On the generality of the laws of learning. Psychological Review, 1970, 77, 400-418.

Tolman, E. C., \& Honzik, C. H. Introduction and removal of reward, and maze performance in rats. University of California Publications in Psychology, 1930, 4, 257-275.

(Received for publication May 31, 1977.) 